JOURNAL OF SYNCHROTRON RADIATION

ISSN 1600-5775

Received 6 April 2015

Accepted 12 October 2015

Edited by J. F. van der Veen

Keywords: laser slicing; femtosecond X-ray pulses; picosecond $\mathrm{X}$-ray pulses; $\mathrm{X}$-ray pulse compression; X-ray switch.

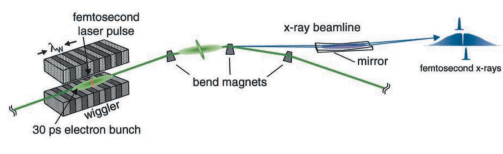

C 2016 International Union of Crystallography

\section{Short X-ray pulses from third-generation light sources}

\author{
A. G. Stepanov ${ }^{a}$ and C. P. Hauri ${ }^{a, b *}$ \\ a Paul Scherrer Institute, 5232 Villigen, Switzerland, and ${ }^{\mathbf{b}}$ Ecole Polytechnique Fédérale de Lausanne, \\ 1015 Lausanne, Switzerland. *Correspondence e-mail: christoph.hauri@psi.ch
}

High-brightness X-ray radiation produced by third-generation synchrotron light sources (TGLS) has been used for numerous time-resolved investigations in many different scientific fields. The typical time duration of X-ray pulses delivered by these large-scale machines is about 50-100 ps. A growing number of time-resolved studies would benefit from X-ray pulses with two or three orders of magnitude shorter duration. Here, techniques explored in the past for shorter X-ray pulse emission at TGLS are reviewed and the perspective towards the realisation of picosecond and sub-picosecond X-ray pulses are discussed.

\section{Introduction}

Characteristic time scales of numerous physical elementary processes, including bond stretching, electronic charge transfer, crystal lattice vibration and electron-phonon interaction are in the range $\sim 0.01-1$ ps. Experimental observation of those processes requires time-resolved techniques with a sub-picosecond resolution. This time resolution can be routinely achieved with pump-probe techniques by means of femtosecond pulses in the optical regime, and a large amount of ultrafast dynamics have been studied this way during the last 30 years (see, for example, the proceedings of the International Conference on Ultrafast Phenomena I-XIX). Such investigations have gained even more in importance with the advent of ultrashort sources in the hard X-ray range since the (sub-) picosecond X-ray pulses offer in addition an excellent spatial resolution down to atomic resolution. In the last two or three decades X-ray synchrotron facilities have become important workhorses for time-resolved investigation with close to atomic resolution and ideas to push their performance towards shorter and shorter X-ray pulses have been raised starting about 20 years ago.

Modern electron accelerator-based X-ray facilities at the large scale can be divided into third- and fourth-generation light sources (Mobilio et al., 2015). The third-generation synchrotron light sources (TGLS) provide X-ray radiation emitted by electron bunches traveling at relativistic speed through insertion devices like wigglers and undulators placed in a storage ring. These facilities aim at high repetitive probing of ultrafast processes at hundreds of $\mathrm{MHz}$ repetition rate and support synchronous data acquisition at the numerous endstations attached to the storage ring. Presently there are about 50 TGLS $^{1}$ worldwide in service, each of them with up to 50 beamlines. Thereby the global community of synchrotron

${ }^{1}$ Lightsources of the World; http://www.lightsources.org/regions. 
users consists of several thousands of scientists and engineers. TGLS provide high-brightness [peak brightness up to $10^{24}$ photons s${ }^{-1} \mathrm{~mm}^{-2} \operatorname{mrad}^{-2}(0.01 \% \text { bandwidth })^{-1}$ ], wavelength-tunable pulses in the soft and hard X-ray range with typical durations of 50-100 ps.

In view of the limited brightness and the still relatively long pulse duration available at TGLS, the technology has advanced meanwhile towards X-ray free-electron lasers (FELs), the fourth-generation light sources. Thanks to a highenergy $(\mathrm{GeV})$ electron linear accelerator followed by a significantly extended undulator section, these FELs provide $\mathrm{X}$-ray pulses with much shorter pulse duration $(\simeq 1-100 \mathrm{fs})$ at much higher peak brightness [up to $10^{33}$ photons $\mathrm{s}^{-1} \mathrm{~mm}^{-2}$ $\left.\operatorname{mrad}^{-2}(0.01 \% \text { bandwidth })^{-1}\right]$ compared with TGLS. Presently there are two hard X-ray FELs in operation worldwide, one in the USA (LCLS) and one in Japan (SACLA), as well as two soft X-ray FELs [Germany (FLASH) and Italy (FERMI@Elettra)]. Several other hard X-ray facilities are under construction such as SwissFEL (Switzerland), the European XFEL (Germany) and PAL-FEL (South Korea). Due to the limited number of running FELs the large demand for beam time can hardly ever be fully covered. Different to TGLS the FEL X-ray beam is allocated to one single experiment at a time and the FEL repetition rate is significantly lower (typically 10-100 Hz). Practically this leads to the implication that a significant number of investigations foreseen at FELs perform pilot studies at high-repetition-rate TGLS prior to FEL beam times. In this view TGLS running with pulse durations similar to a FEL would be particularly beneficial but also other X-ray studies employing time-domain spectroscopy would profit from such developments at TGLS. We mention that a large class of time-resolved experiments in principle do not need the ultrashort (<50 fs) bright X-ray pulses provided by FELs but could deal with somewhat longer ( 0.1-10 ps) pulses. Bridging this gap between TGLS and FELs includes developments towards brighter and shorter TGLS X-ray pulses with enhanced coherence. This will open novel science opportunities as these sources will be complementary to the presently available large-scale facilities.

In this paper we review the existing techniques for picosecond and sub-picosecond X-ray pulse generation at TGLS and discuss concepts and ideas which could potentially be used for this purpose. The manuscript is organized as follows. In $\S 2$ the laser-based slicing method is reviewed. $\$ 3$ describes briefly the formation of picosecond X-ray pulses from TGLS operating in the lowalpha mode and will describe other trends to improve TGLS coherence and pulse length properties. In $\$ 4$ we consider different types of ultrafast Xray switches. $\$ 5$ reviews some new approaches for efficient compression of $\mathrm{X}$-ray pulses while $\S 6$ gives an outlook on future use of TGLS in the era of FELs. While the methods presented in $\$ 2$ and $\$ 3$ rely mainly on shaping the electron beam before sending it through a radiative element, the methods discussed in $\S 4$ and $\S 5$ aim at manipulating the $\mathrm{X}$-ray pulse shape directly.

\section{Laser slicing method}

In 1996, A. A. Zholents and M. S. Zolotorev proposed the use of interaction of femtosecond laser pulses with electrons in a storage ring to produce femtosecond X-ray pulses of synchrotron radiation (Zholents \& Zolotorev, 1996). The interaction of electrons with the intense laser pulse $(\sim 1-2 \mathrm{~mJ}$, $50-100 \mathrm{fs}, 1-6 \mathrm{kHz}$ ) takes place in a wiggler structure (Fig. 1) and leads to a modulation of electron energy within a thin slice of the electron bunch. The modulation of electron energy leads to a spatial separation from the main bunch by means of a bending magnet in the storage ring and is then used to generate femtosecond X-rays at another bending magnet (or wiggler). The background contribution from the remaining electrons in the long bunch can be reduced by placing an aperture at an image plane of the source (created by the beamline optics) to select only the short X-ray pulses originating from the sliced electrons. This radiation has approximately the same duration as the femtosecond laser pulse. The generation of femtosecond pulses of synchrotron radiation by this technique was first demonstrated at the Advanced Light Source (ALS) of Lawrence Berkeley National Laboratory in the USA (Schoenlein et al., 2000). The concept of slicing provides femtosecond X-ray pulses which are naturally synchronized to the optical laser. The scheme offers thus great opportunities for ultrafast optical pump/X-ray probe experiments as the intrinsic jitter between the X-ray and optical pulse is a small fraction of the pulse duration.

Direct measurement of the femtosecond hard X-ray pulses from a slicing source has been difficult in the past as a promising technique based on laser-streaking has only been developed recently for hard X-rays (Frühling et al., 2009; Juranić et al., 2014). Alternatively, cross-correlation measurements have been performed between an optical $50 \mathrm{fs}$ laser pulse and visible $(\sim 2 \mathrm{eV})$ synchrotron pulse generated by the laser slicing technique and the results are shown in Fig. 2. Visible synchrotron radiation is employed as the time structure of the electron bunch spontaneous emission is invariant over its entire frequency spectrum. According to these measurements the synchrotron pulse has a duration of about 150 fs. It was assumed that the $\mathrm{X}$-ray pulse duration is 


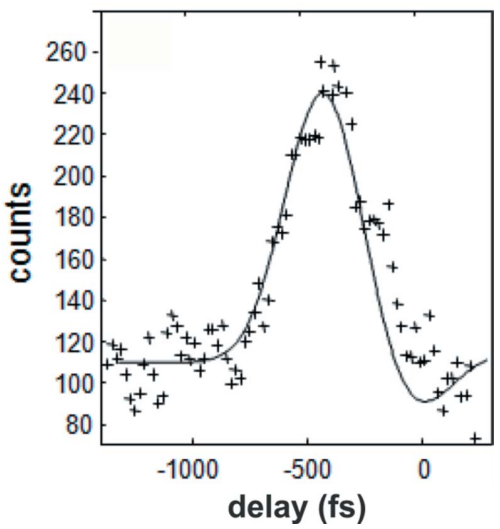

Figure 2

Cross-correlation measurement between an optical laser pulse and visible synchrotron radiation generated by the laser slicing technique. From Schoenlein et al. (2000), reprinted with permission from AAAS.

approximately equal to the pulse duration of the optical femtosecond laser pulse.

Apart from at the ALS the slicing technique has been successfully implemented at storage-ring-based synchrotron radiation sources in Germany (BESSY II; Khan et al., 2006) and Switzerland (SLS) and will be soon operational at the synchrotron radiation facility Soleil in France. The laser slicing facility at BESSY II provides $\sim 100 \mathrm{fs}$ soft $\mathrm{X}$-ray pulses in the range $250-1400 \mathrm{eV}$ with an average flux of $\sim 10^{6}$ photons s $\mathrm{s}^{-1}$ $(0.1 \% \text { bandwidth })^{-1}$ (Holldack et al., 2014). The first tunable femtosecond hard (2.5-1 А) X-ray source was demonstrated at the Paul Scherrer Institute (Switzerland) in 2007 and provides up to $8 \times 10^{5}$ photons $\mathrm{s}^{-1}$ in a $1.2 \%$ bandwidth (Beaud et al., 2007). Femtosecond X-ray pulses obtained by this technique were used in a number of time-resolved studies, for example for the observation of the coherent phononpolaritons in ferroelectric lithium tantalite crystal (Cavalleri et al., 2006), coherent phonons in bismuth (Johnson et al., 2008), light-induced spin crossover dynamics in an iron(II) complex (Bressler et al., 2009), ultrafast spin and demagnetization dynamics (Bergead et al., 2014; Eschenlohr et al., 2013; Wietstruk et al., 2011; Radu et al., 2011; Boeglin et al., 2010; Stamm et al., 2007) and many more (Huse et al., 2011; Rettig et al., 2015; Mariager et al., 2014).

Application of the laser slicing technique results in a dramatic reduction (by a factor of $10^{8}$ ) of the average X-ray source brightness compared with the normal TGLS operation mode. This mainly happens because only a small fraction of the bunch electrons participate in the generation of the ultrashort X-ray pulse and due to the repetition rate of the optical slicing laser $(1-6 \mathrm{kHz})$ which is significantly lower than the repetition rate of the synchrotron radiation facility. To overcome this problem, Zholents et al. proposed another technique where pulses of synchrotron radiation much shorter than a picosecond can be obtained without a reduction of the electron bunch length (Zholents et al., 1999). In their approach the electron bunch is tilted by an RF deflector cavity. Simultaneously the same technique was propsed by Katoh (1999). In the undulator, each sub-picosecond slice of the electron bunch emits radiation in a solid angle which slightly differs from the neighboring slices. The linear correlation between the longitudinal coordinates of the electrons within the electron bunch and their emitting angle gives rise to an X-ray pulse with a linearly tilted pulse front. A few-picosecond pulse duration can be achieved after the undulator by compensating the pulse front tilt of the emitted X-ray pulse by means of a pair of asymmetrically cut silicon crystals $\mathrm{Si}(220)$. Numerical modeling shows that a compression factor of up to 100 is achievable. As the technical implementation in a storage ring seems challenging, an experimental realization of this technique has not been carried out so far.

A recent proposal by $\mathrm{He}$ et al. discusses another method to generate ultrashort X-ray pulses based on electron bunch slicing. The main idea is to cross an electron bunch circulating in a synchrotron radiation storage ring with a low-energy $20 \mathrm{MeV}$ electron bunch under a specific angle (He et al., 2014). During the interaction a short slice of electrons is kicked from the core of the storage ring electron bunch by the Coulomb force. The numerical study predicts the slice to radiate ultrashort $160 \mathrm{fs} \mathrm{X}$-ray pulses when passing through a suitable undulator. By tuning the electron energy and crossing angle, different X-ray pulse properties could be reached. An experimental verification, however, has not yet been performed.

\section{Low-alpha mode and other trends at TGLS}

The duration of X-ray pulses generated by TGLS is determined by the electron bunch length in first order. For this reason a natural way to decrease the X-ray pulse duration is to use shorter electron bunches. However, storage of short electron bunches in a ring is challenging because of instabilities arising from bunch-induced wake fields among other reasons (Limborg, 1998). One way to shorten the electron bunch is by decreasing the momentum compaction factor using low-alpha optics in the storage ring. Generation of subpicosecond electron bunches with a long lifetime by this lowalpha method was first demonstrated at BESSY II in 2002 (Abo-Bakr et al., 2002). The method goes along with a dramatic reduction in the electron bunch charge and a modified filling pattern, which simultaneously decreases the average current and the X-ray flux at all endstations at the storage ring. Operating TGLS in the low-alpha mode leads in addition to reduced storage ring stability and practically excludes synchronous operation of short-pulse and photondemanding experiments.

The X-ray pulses of $1-10 \mathrm{ps}$ provided by the low-alpha mode are about 20 times longer than those provided by femtoslicing sources (see \$2). However, the low-alpha mode has in principle the advantage that the average photon flux and bunch repetition rate $(1-500 \mathrm{MHz})$ are significantly higher than provided by the femto-slicing technique. Several TGLS facilities offer thus the low-alpha mode to users including Soleil (France), BESSY (Germany), SSRL (USA), SLS (Switzerland), DLS (UK) on an irregular base. Due to the time-consuming setting up and the severe impact on photondemanding applications, the operation mode may be limited to 
a few days per year. We mention that running the storage ring in the low-alpha mode is in principle favorable for the $\mathrm{THz}$ user community as the $\mathrm{THz}$ emission by coherent synchrotron radiation is much higher for short picosecond electron bunches (Abo-Bakr et al., 2003; Holldack et al., 2006; Byrd et al., 2006).

An important trend for future synchrotron sources is to provide variable pulse length at high average beam brilliance and flux. A significant improvement in peak brilliance is expected by employing a superconducting radiofrequency cavity scheme (Huang et al., 2014). Recently a conceptual design study of a variable pulse length storage ring (VSR) has been presented for the BESSY facility (BESSY VSR). The source shall provide simultaneous operation of long (approximately $15 \mathrm{ps}$ r.m.s.) and short (approximately $1.7 \mathrm{ps}$ r.m.s.) pulses at a flux similar to the standard operation mode of a synchrotron source with a large bunch charge of $640 \mathrm{pC}$ and at the full RF repetition rate $(500 \mathrm{MHz})$. Even shorter pulses (300 fs r.m.s.) shall be provided at a reduced bunch charge (30 pC) (Wuestenfeld et al., 2011). The availability of such high-flux and picosecond-pulse beams will be beneficial for a large user community as the X-ray source parameters are complementary to what is provided by high-peak-brilliance low-repetition-rate femtosecond free-electron lasers (FELs) and standard synchrotron radiation facilities.

Finally we mention that recent progress in storage ring design will pave the way towards low-emittance synchrotron facilities with increased brightness and coherence properties. Presently several TGLS facilities are realising diffractionlimited storage rings (DLSR) (e.g. MAX-IV) or are considering an upgrade towards DLSR (SLS, ESRF, SPring-8, Soleil, ALS) which will provide an ultimate small transverse emittance. However, while focusing and coherence will be improved, the X-ray pulse duration of DLSR is not expected to be significantly shorter than what is provided by typical

(a)

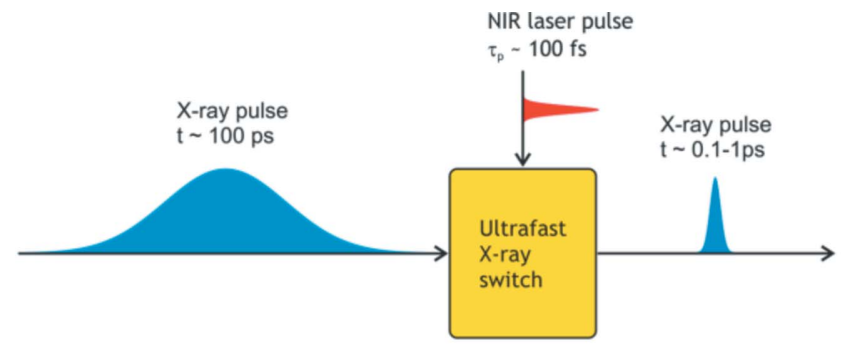

(b)

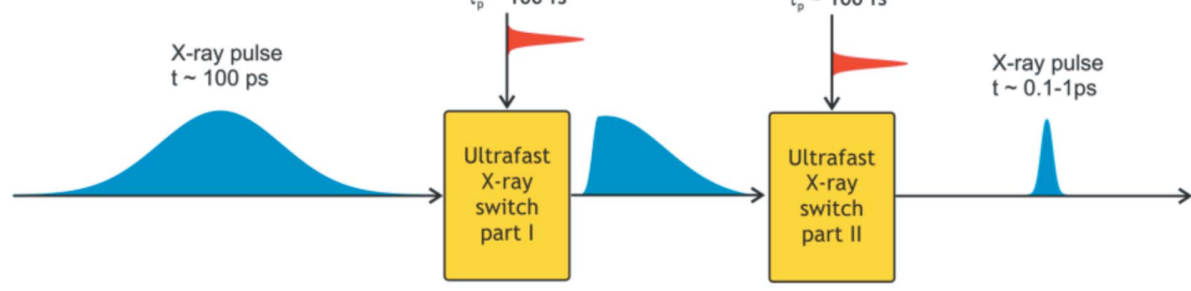

Figure 3

Basic operation principle of ultrafast X-ray switches. (a) A single femtosecond NIR laser pulse is used for the switch opening and closing. (b) The first NIR laser pulse (I) leads to the switch opening, the second NIR laser pulse (II) provides the switch closing. third-generation synchrotron sources. Even though the concept of DLSR employing low-dispersive magnetic beam steering may in principle favor short pulse generation, the present designs aim for longer pulses in order to avoid beam instabilities by high impedance issues caused by orbiting short bunches in tight vacuum chambers. Nevertheless, DLSR sources are expected to be highly beneficial for scientific applications like coherent diffractive imaging, ptychography, spectroscopic nanoprobes and others where the ultimate timeresolution is not required. A summary on DLSR designs and potential science applications can be found by Hettel (2014) and Eriksson et al. (2014), respectively.

\section{X-ray switches}

Several types of ultrafast switches have been proposed for shortening the synchrotron X-ray pulse. Compared with the techniques presented above, the X-ray switches act directly on the X-ray pulse rather than the electron bunch. The basic operation principle of an X-ray switch is illustrated in Fig. 3(a). Most of these switches require near-infrared (NIR) femtosecond laser pulses as a gate which introduces a fast laserinduced change of the X-ray diffraction properties. X-ray and femtosecond NIR laser pulses arrive simultaneously at the switch. Before the femtosecond laser pulse arrives at the switch, no X-ray radiation is transmitted, i.e. the switch is closed. The NIR laser pulse leads to an ultrafast switch opening which allows the X-ray radiation to cross the switch. After that, two scenarios can be realised: the switch closes by itself as soon as femtosecond laser radiation vanishes (Fig. 3a) or a second femtosecond laser pulse closes the switch (Fig. 3b). Up to now most of the demonstrated and proposed X-ray switches are based on X-ray Bragg diffraction which is modulated by the femtosecond laser pulse; however, some other switching mechanisms can be applied as well.

To the best of our knowledge the first attempt at shortening TGLS X-ray pulses of $\sim 100 \mathrm{ps}$ was reported in 1998 (Larsson et al., 1998). In this experiment the X-ray Bragg diffraction from a InSb crystal was modulated by the crystal transient disordering induced by a femtosecond laser pulse. In this way X-ray pulses of about $\sim 50 \mathrm{ps}$ with short back slopes $(\sim 1 \mathrm{ps})$ were deflected in a different solid angle which could be separated from the main pulse. The use of these pulses in time-resolved X-ray diffraction measurements provided a time resolution of less than 2 ps.

In 1999 Bucksbaum and Merlin proposed a scheme based on a phonon Bragg diffraction switch for hard X-ray radiation (Bucksbaum \& Merlin, 1999), where a superlattice of optical phonons is used for deflecting the X-ray pulse. A schematic of their proposed phonon 


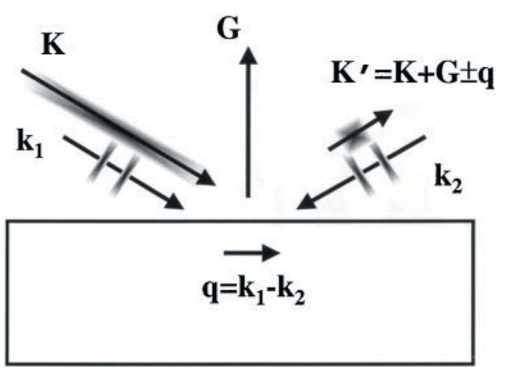

Figure 4

Schematic of the phonon Bragg switch. $\mathbf{G}$ is a reciprocal lattice vector in the crystal; $\mathbf{k}_{1}$ and $\mathbf{k}_{2}$ are wavevectors of femtosecond laser pulses creating phonon superlattice with wavevector $\mathbf{q}$. $\mathbf{K}$ and $\mathbf{K}^{\prime}$ are wavevectors of incident and diffracted X-ray pulses. Reprinted from Bucksbaum \& Merlin (1999), Copyright (1999), with permission from Elsevier.

Bragg switch is shown in Fig. 4. The transient superlattice is generated by two ultrafast laser pulses interfering at the crystal surface. We mention that the authors proposed to apply two pairs of femtosecond laser pulses with a relative delay to each other. The first pair creates the phonon superlattice and the second pair rubs it out in a coherent control way. In a follow-up publication more detailed calculations on the phonon Bragg diffraction switch have been presented (Sheppard et al., 2005). It was concluded that very large phonon amplitudes are necessary for achieving only a few percent Xray peak reflectivity in the sidebands related to the phonon superlattice. From these numerical studies it was concluded that it is very likely that such large oscillations would result in the crystal melting.

Later, a traveling wave interaction scheme was suggested for the phonon Bragg X-ray switch (Nazarkin et al., 2004). In this scheme the crystal should be transparent for both X-ray and laser radiation. Due to a long interaction length between the X-ray and laser-induced lattice vibrations this scheme is expected to provide significant increase in the switching efficiency.

A piezoelectric sub-nanosecond switch based on diffraction was reported in 2006 (Grigoriev et al., 2006). This switch mainly aimed at extracting an individual $\sim 100 \mathrm{ps} \mathrm{X}$-ray pulse at up to a $\mathrm{kHz}$ repetition rate from the $\mathrm{MHz}$ pulse train offered by the synchrotron source. The piezoelectric switch consists of a $300 \mathrm{~nm}$-thick film of $\mathrm{Pb}(\mathrm{Zr}, \mathrm{Ti}) \mathrm{O}_{3}$, in the tetragonal crystal phase, between $100 \mathrm{~nm}$-thick conducting $\mathrm{SrRuO}_{3}$ electrodes. The switch was triggered by electrical pulses of $15 \mathrm{~V}$ amplitude and $10 \mathrm{~ns}$ duration. The (002) Bragg reflection intensity as a function of scattering angle for three different time positions of the electrical pulse relative to the X-ray pulse is shown in Fig. 5.

The effectiveness of the switch is plotted in Fig. 6 and quantified by the intensity ratio $I_{\text {passing }} / I_{\text {blocking }}$ between the passing and blocking operation modes. This switch has an opening time of $600 \mathrm{ps}$, which is about two times longer than the electric pulse slope duration ( $300 \mathrm{ps}$ ). The authors suppose that this is due to a combination of the jitter in the timing circuit and the impedance mismatch between the capacitive
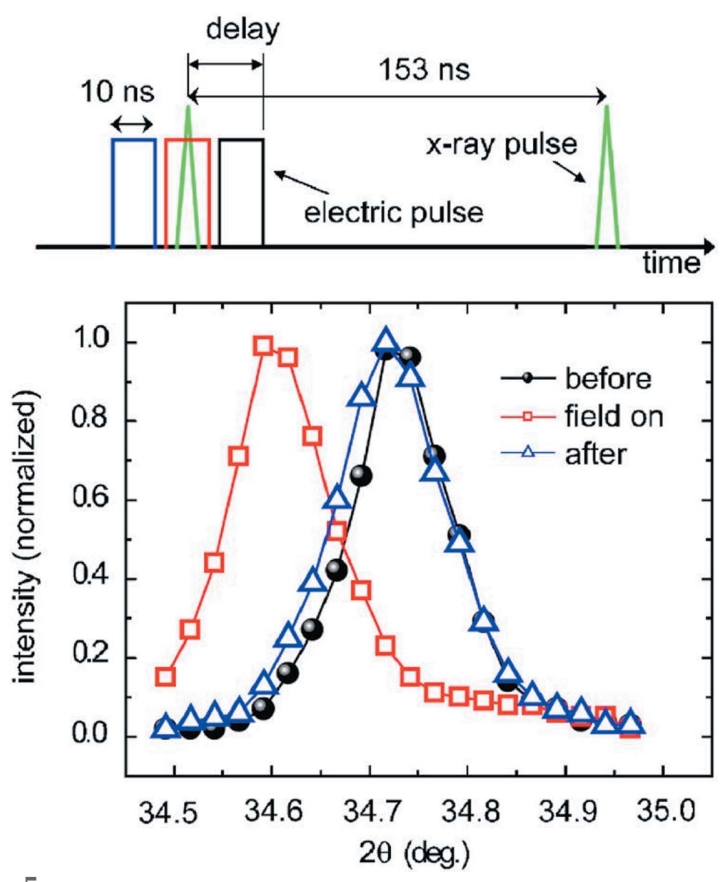

Figure 5

The (002) Bragg reflection as a function of scattering angle for three different time positions of the electrical pulse relative to the probing $\mathrm{X}$-ray pulse. The timing diagram is shown in the upper part of the figure. Reprinted with permission from Grigoriev et al. (2006), Copyright 2006, AIP Publishing LCC.

load and the coaxial connection between the switch and the pulse generator.

We speculate that a faster switching time could be achieved by using shorter electric pulses. For instance, recent progress in the generation of single-cycle $\mathrm{THz}$ pulses allows a peak field strength up to a few $\mathrm{MV} \mathrm{cm}^{-1}$ to be obtained with a rise time of about 1 ps (Stepanov et al., 2010) or even shorter (Vicario et al., 2014a, 2015). The electrical field transient of the singlecycle $\mathrm{THz}$ pulse could in principle replace the external voltage switch used above. However, determination of the shortest triggering time which can be reached with $\mathrm{THz}-$ driven piezoelectric switches requires additional experimental investigations.

Recently, switching of the hard X-ray Bragg reflectivity of a $\mathrm{SrRuO}_{3} / \mathrm{SrTiO}_{3}$ superlattice (Herzog et al., 2010) and a $\mathrm{SrRuO}_{3} / \mathrm{SrTiO}_{3}$ double layer structure (Gaal et al., 2012;

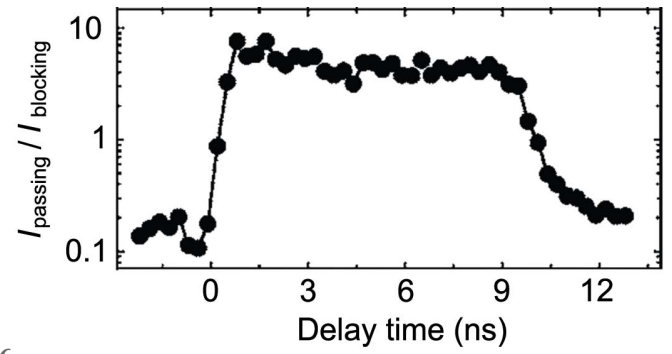

Figure 6

The intensity ratio $I_{\text {passing }} / I_{\text {blocking }}$ between the passing and blocking operation modes. With a response time of less than $1 \mathrm{~ns}$ the piezoelectric switch by Grigoriev et al. (2006) is able to isolate any single synchrotron $\mathrm{X}$-ray pulse at existing synchrotron sources. Reprinted with permission from Grigoriev et al. (2006). Copyright 2006, AIP Publishing LCC. 

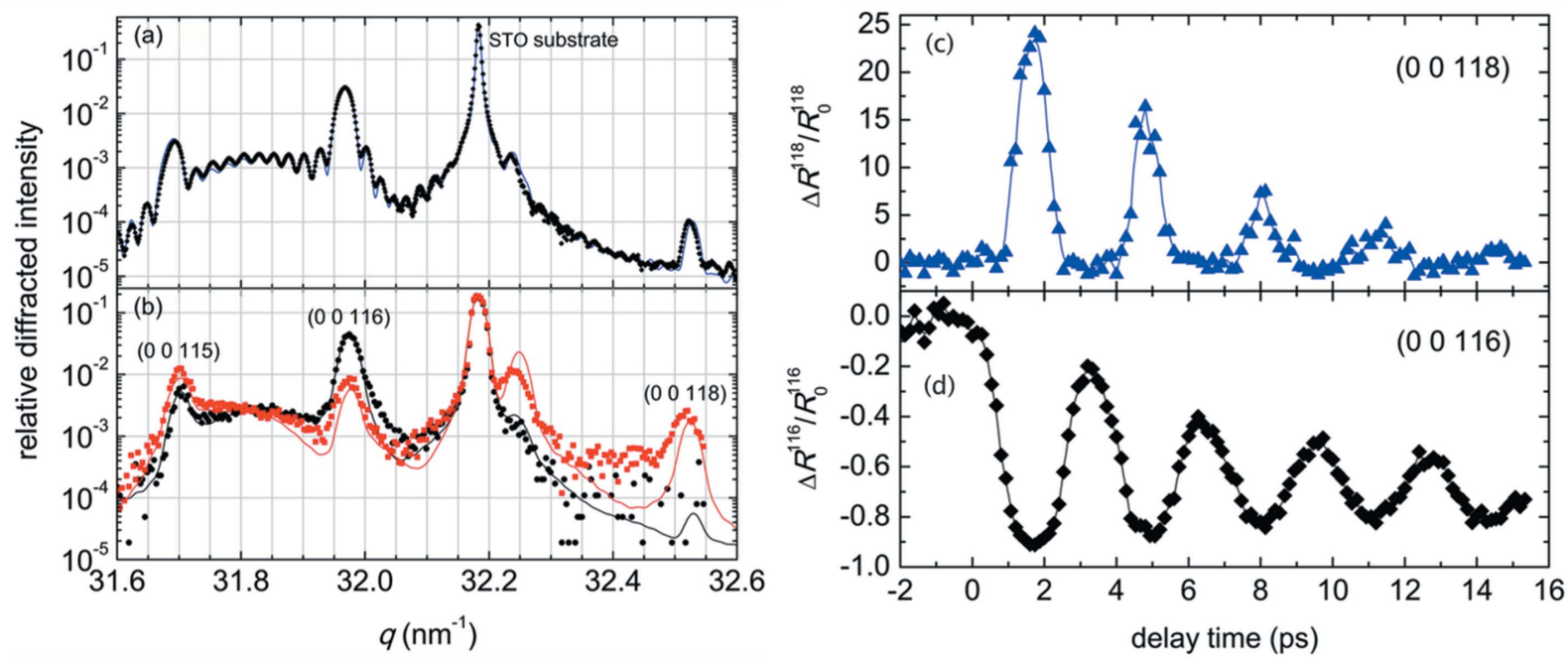

Figure 7

(a) Black diamonds: static rocking curve recorded at BESSY II. (b) Rocking curve of the unexcited SRO/STO superlattice (black bullets) and 1.6 ps after laser pulse excitation (red squares). These data sets were measured at the SLS in laser slicing mode. ( $c, d$ ) Relative change of the transient X-ray Bragg

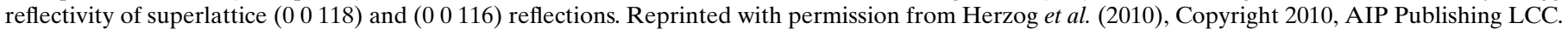

Freund \& Levine, 1970) excited by femtosecond NIR laser pulse was demonstrated. Absorption of the femtosecond pump pulse in a thin $(15 \mathrm{~nm})$ metallic $\mathrm{SrRuO}_{3}$ layer induced a fast expansion of the layer, which changed the Bragg diffraction intensity. $\mathrm{SrTiO}_{3}$ layers transparent to NIR laser pulses are mainly applied for providing fast heat flow and return of the $\mathrm{SrRuO}_{3}$ layers to the initial, not expanded, state. Perfect matching of the acoustic impedances of $\mathrm{SrRuO}_{3}$ and $\mathrm{SrTiO}_{3}$ diminishes acoustic wave reflection at the interface.

The $\mathrm{SrRuO}_{3} / \mathrm{SrTiO}_{3}$ superlattice switching was tested with the laser slicing beamline of the Swiss Light Source providing $\mathrm{X}$-ray pulses of $140 \mathrm{fs}$ in duration at a photon energy of $5.2 \mathrm{keV}$ and at a mean photon number of 20 photons per X-ray pulse. The experimental results are shown in Figs. $7(a)-7(d)$.

Oscillations in Bragg reflectivity [Figs. $7(c)-7(d)$ ] appear due to acoustic phonon wave propagation in the $\mathrm{SrRuO}_{3}$ / $\mathrm{SrTiO}_{3}$ superlattice. The oscillations were suppressed in the case of a $\mathrm{SrRuO}_{3} / \mathrm{SrTiO}_{3}$ double layer structure (Herzog et al., 2010; Gaal et al., 2012). This double layer switch was verified with a laser-plasma source of femtosecond hard X-ray pulses

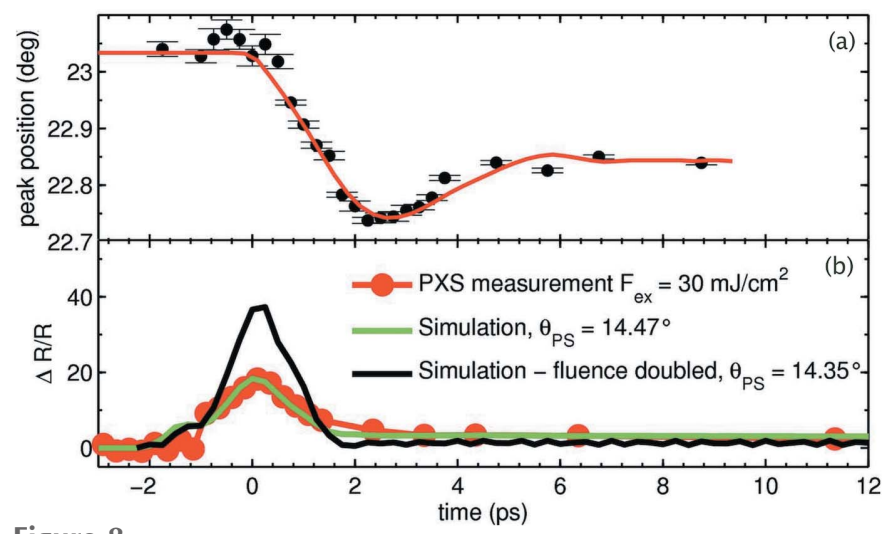

Figure 8

(a) Transient shift of the diffraction peak measured with the laser-plasma X-ray source. (b) Measurements (red bullets) and simulations (green and black solid lines) of switching. From Gaal et al. (2014). with a photon energy of $8.047 \mathrm{kV}$. Results of these measurements are shown in Fig. 8.

The switch was tested with an X-ray synchrotron source (ESRF) as well. The maximum diffraction efficiency achieved with this switch is about $10^{-3}$. The synchrotron probe pulse was shortened to approximately 2 ps.

Shorter X-ray pulses can be obtained by a parametric nonlinear mixing of the X-ray synchrotron and optical waves. $\mathrm{X}$-ray and optical wave nonlinear mixing, specifically sumfrequency generation (SFG), have been considered for a long time (Freund \& Levine, 1970). Recently the first experimental observation of X-ray and optical sum frequency generation was reported (Glover et al., 2012). A sketch of the experimental layout and main results are summarized in Fig. 9. The principle has been tested with an $8 \mathrm{kV} \sim 80 \mathrm{fs}$ X-ray pulse from a FEL together with a 2 ps NIR laser pulse at an intensity of $10^{10} \mathrm{~W} \mathrm{~cm}^{-2}$ using diamond as a nonlinear medium. The measured energy conversion efficiency of $3 \times 10^{-7}$ corresponds to a nonlinear susceptibility of $<1.6 \times 10^{-14}$ e.s.u. From the publication it is not clear whether the efficiency of the sum frequency generation is limited by the absorption of X-ray and optical waves or by the velocity mismatch in the diamond plate. The authors claim that numerical estimations suggest the conversion efficiency to reach up to $10^{-3}$ by overcoming these two obstacles and by optimizing X-ray radiation parameters (divergence and monochromaticity). Such high conversion efficiency would be very promising for an ultrafast $\mathrm{X}$-ray switch. However, to our mind it is not clear how phasematching between X-ray and NIR radiation could be achieved efficiently.

Another nonlinear process which is of potential interest for shaping and switching an X-ray pulse is the electromagnetically induced transparency (EIT). EIT can be illustrated in a $\Lambda$-type medium characterized by atomic levels $|1\rangle,|2\rangle$ and $|3\rangle$ with energies $E_{1}>E_{2}>E_{3}$ (Fig. 10). In such a medium the resonant absorption on the $|3\rangle \rightarrow|1\rangle$ transition can be strongly suppressed by simultaneously irradiating the 

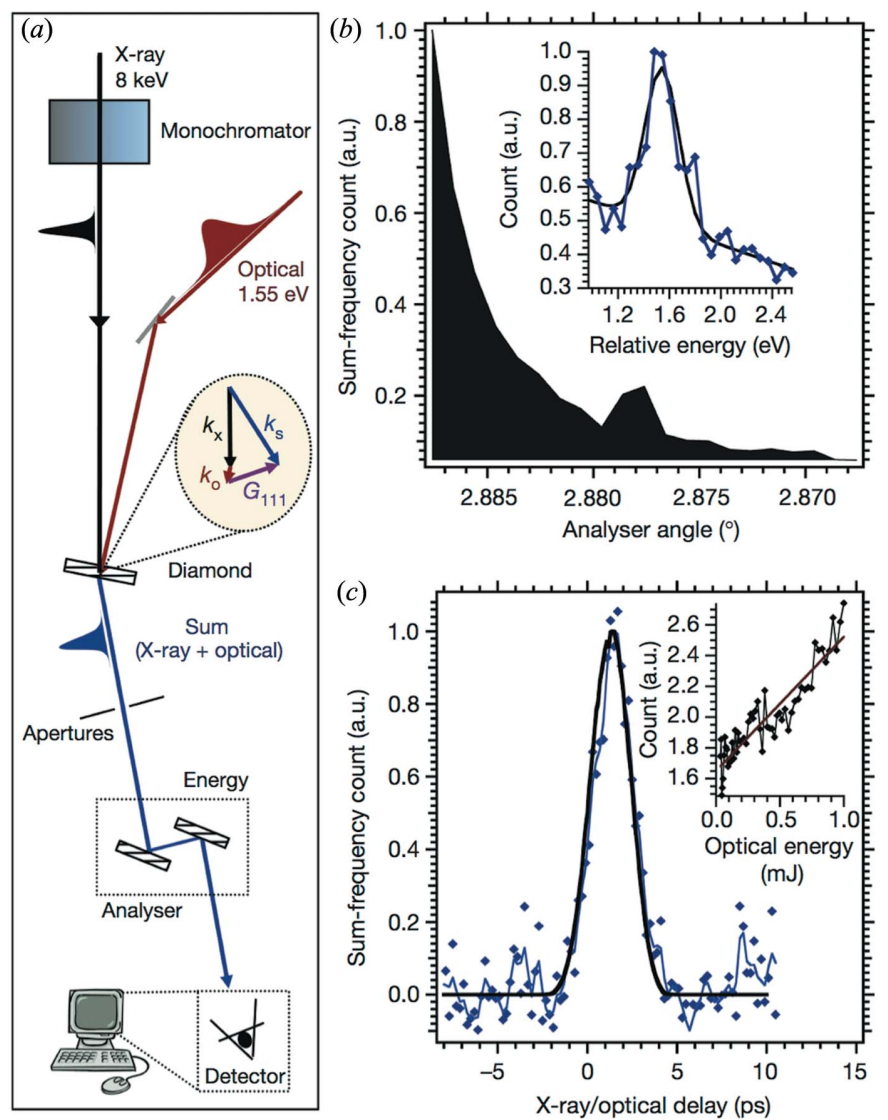

Figure 9

(a) Experimental layout for X-ray $(8 \mathrm{keV})$ and optical $(1.55 \mathrm{eV})$ sum frequency generation (SFG) in diamond. (b) X-ray signal versus energy analyzer angle. Inset: energy relative to $8 \mathrm{keV}$. The SFG energy exceeds the X-ray energy by one optical photon. (c) SFG signal versus X-ray/ optical time delay. Black cross-correlation curve ( $2.5 \mathrm{ps}$ full-width at halfmaximum) solution of the wave equation for an $80 \mathrm{fs} \mathrm{X}$-ray pulse and a 1.7 ps optical pulse. Inset: SFG signal versus optical intensity. The red line is a fit to a linear dependence on optical intensity. Reprinted by permission from Macmillan Publishers Ltd: Nature (Glover et al., 2012), Copyright (2012).

medium with an intense laser pulse with a photon energy close to the $|2\rangle \rightarrow|1\rangle$ transition. The theory behind EIT for creation of an X-ray switch was first described by Buth and co-workers in 2007 (Buth et al., 2007). The performed numerical studies with $\mathrm{Ne}$ gas showed a dramatic change of the photoabsorption cross section in the vicinity of the $1 s \rightarrow 3 p$ resonance $(867.4 \mathrm{eV})$ upon illumination with a laser pulse at an intensity of $10^{13} \mathrm{~W} \mathrm{~cm}^{-2}$. The method is, in principle, suitable for imprinting pulse shapes of the optical dressing pulse onto the $\mathrm{X}$-ray radiation or to slice short $\mathrm{X}$-ray pulses from a longer one. Indeed, Buth and co-workers suggested to generate two ultrashort X-ray pulses by EIT by overlapping a 100 ps-long $\mathrm{X}$-ray pulse with two intense few-ps-long laser pulses in Ne. The time delay between the two X-ray pulses can be controlled by changing the time delay between the two laser dressing pulses. Such a scheme opens an avenue towards ultrafast X-ray pump/X-ray probe experiments.

While an experimental verification at hard X-ray energies has not been performed so far, the concept of EIT has been demonstrated recently in the VUV range by means of a table-
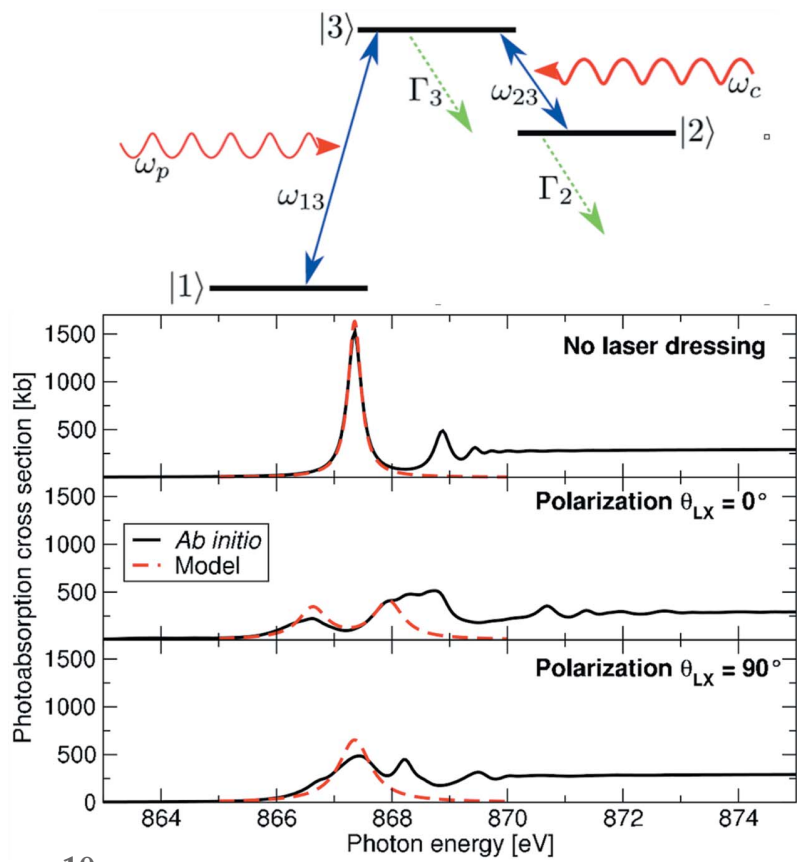

Figure 10

(Top) Schematic illustrating electromagnetically induced transparency (EIT). (Bottom) Results of ab initio (black solid lines) and a three-level model (red dashed lines) calculations of X-ray absorption cross section of neon near the $K$-edge with and without laser dressing, respectively. $\theta_{\mathrm{LX}}$ is the angle between the polarization vectors of the laser and the X-rays. The laser operates at a wavelength of $800 \mathrm{~nm}$ and at an intensity of $10^{13} \mathrm{~W} \mathrm{~cm}^{-2}$. Reprinted with permission from Buth et al. (2007), Copyright (2007) by the American Physical Society.

top HHG source. In 2008 an ultrafast variation of the absorption in the XUV range $(60.15 \mathrm{eV})$ was reported based on the EIT of the $1 s^{2} \rightarrow 2 s 2 p$ transition in He irradiated by intense femtosecond laser pulses (Loh et al., 2008). The set-up used in those studies and the main results are shown in Figs. 11 and 12 , respectively.

A few years later, EIT has been transferred to the soft X-ray region and experimental results were obtained using the synchrotron radiation source at ALS (Glover et al., 2010). Similar to the HHG setup the X-ray and laser NIR pulses copropagated through the neon gas cell (Fig. 13). In that experiment EIT was employed to characterize the femtosecond X-ray pulses of the ALS slicing source (Glover et al.,

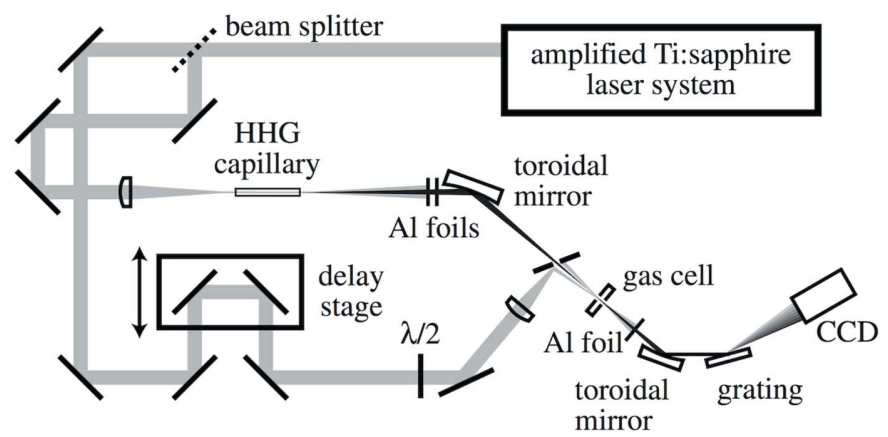

Figure 11

Experimental set-up used for observation of ultrafast electromagnetically induced transparency in the XUV spectral region. Reprinted from Loh et al. (2008), Copyright (2008), with permission from Elsevier. 


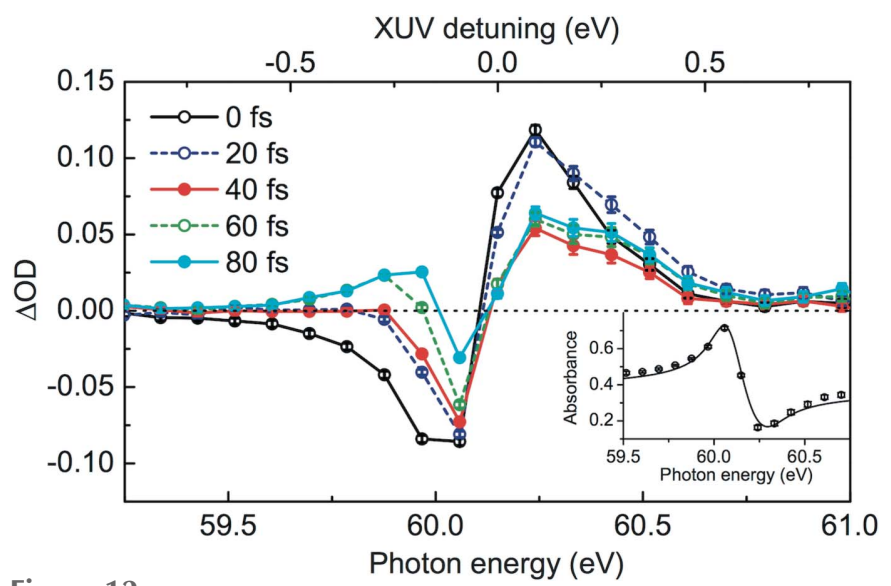

Figure 12

Transient absorption spectra measured at different dressing-probe time delays. The static (field-free) absorption spectrum for the $1 s^{2} \rightarrow 2 s 2 p$ transition is shown in the inset. Reprinted from Loh et al. (2008), Copyright (2008), with permission from Elsevier.

2010) but X-ray pulse shaping has been proposed to achieve spectral and temporal control by using multiple optical control pulses.

The observed transient increase in transmission by a factor of about 3 at $867.1 \mathrm{eV}$ (Fig. 14) is suitable for an ultrafast $\mathrm{X}$-ray switch. A disadvantage of this approach is the very narrow spectral region of switching (about $0.5 \mathrm{eV}$ ). An extension of this technique to the hard X-ray region was suggested by employing rare gas ions (Young et al., 2010a). This approach, however, requires much higher laser intensity and has not been experimentally demonstrated so far.

The X-ray ultrafast switch comes typically at the cost of a reduction of the X-ray average brightness of the TGLS X-ray source. At best (i.e. perfect switching) the reduction factor is dominantly determined by two factors: $k_{1}=T_{\mathrm{L}} / T_{\mathrm{e}}, k_{2}=f_{\mathrm{L}} / f_{\mathrm{B}}$, with $T_{\mathrm{L}}$ and $T_{\mathrm{e}}$ being the laser pulse and electron bunch durations and $f_{\mathrm{L}}$ and $f_{\mathrm{B}}$ the laser and electron bunch repetition rates, respectively. The same factors describe the reduction of the average brightness in the case of the laser slicing technique. In practice one should expect a stronger demagnification related to switcher 'imperfectness'. Smaller reduction of

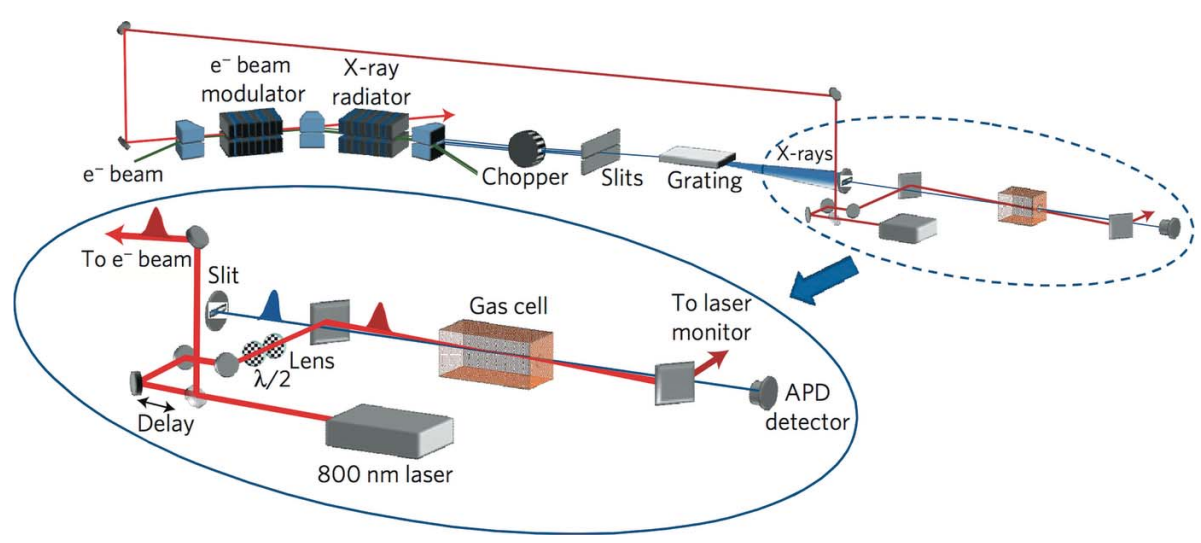

Figure 13

Experimental set-up used for observation of ultrafast EIT in the soft X-ray region. Reprinted by permission from Macmillan Publishers Ltd: Nature (Glover et al., 2010), Copyright (2010).

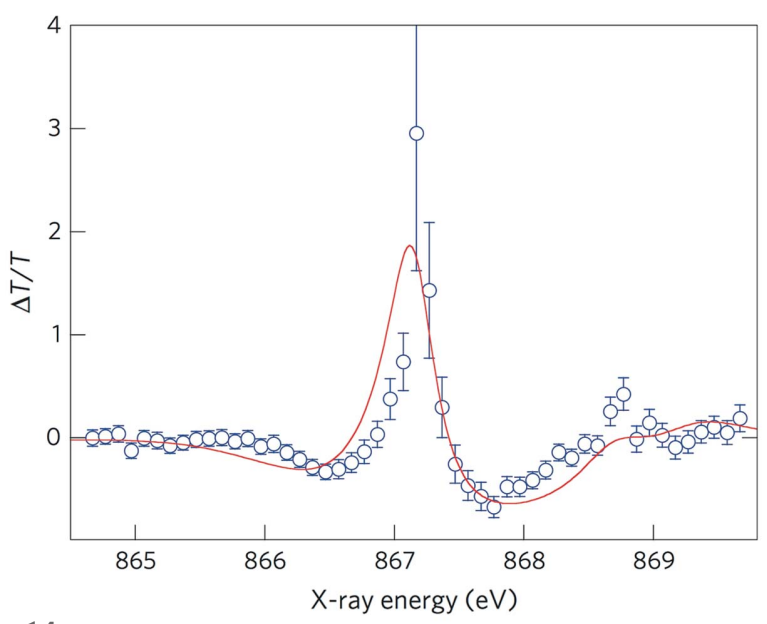

Figure 14

The fractional change in X-ray transmission induced by the coupling laser with a peak intensity of $2.5 \times 10^{13} \mathrm{~W} \mathrm{~cm}^{-2}$ in the parallel polarization configuration. The solid line shows theoretical simulations. Reprinted by permission from Macmillan Publishers Ltd: Nature (Glover et al., 2010), Copyright (2010).

the X-ray average brightness could be achieved by using a compression of the synchrotron pulse instead of cutting it with an ultrafast switch.

We finally mention that recent technological advances in powerful terahertz sources may open novel opportunities for X-ray switches in the future (Shalaby \& Hauri, 2015; Vicario et al., 2014b; Ruchert et al., 2013). As the field strength of these sources approaches the field strength present in atomic and molecular bindings, it becomes feasible to coherently excite strong linear and nonlinear phonon vibrations in crystals directly by the electric field of the THz pulse (Katayama et al., 2012). This would result in Bragg diffraction conditions which are modified on the time scale of the THz field oscillation. The coherent lattice excitation could be terminated by means of a second, delayed, $\mathrm{THz}$ pulse which carries a $180^{\circ}$ phase shift. The expected X-ray pulse duration would be of the order of $0.5-5$ ps depending on the applied $\mathrm{THz}$ carrier frequency.

\section{X-ray compressors}

In view of the quest for bright and short $\mathrm{X}$-ray pulses, a compression technique similar to that routinely employed for optical chirped-pulse amplification laser systems has been used for chirped X-ray pulses. The main idea behind this is to profit from the fact that X-ray pulses from TGLS often carry a longitudinal frequency chirp. This means that the $\mathrm{X}$-ray pulse duration is not as short as it could be according to the time-bandwidth product. X-ray compressors have therefore been proposed and studied in the past to compensate for the residual frequency chirp in order to produce shortest possible X-ray pulses. The chirp 
is typically compensated by an assembly of dispersive elements (e.g. gratings) which rearrange all frequency components in time to arrive at once on target.

$\mathrm{X}$-ray switches reviewed in the previous chapter rely on slicing the X-ray pulse. The compression-based technology discussed here employs all photons, and losses are mainly dominated by the diffraction efficiency of the dispersive elements. A first example for X-ray pulse compression was already given in $\S 2$ which addressed the orbit deflection technique for sub-ps X-ray pulse generation with tilted pulse fronts (Zholents et al., 1999). Another compression approach is based on the use of chirped NIR laser pulses with electron beams in order to generate frequency-chirped electron bunches (Hemsing et al., 2014). The frequency-chirped X-ray pulses generated by these electron bunches can then be compressed in a way similar to that used in chirped pulses amplification of femtosecond laser pulses.

In 2009 the generation of chirped soft X-ray pulses with a tapered undulator and its compression by means of a double grating diffraction was reported (Fujiki et al., 2009). A schematic of this experiment is shown in Fig. 15. Successful compression down to $37 \%$ of the original pulse duration ( $\sim 100 \mathrm{ps}$ ) was achieved. The authors claim that the technique could potentially be used for the generation of even shorter pulses down to the few femtoseconds regime. Practical realisation of this compression approach strongly depends on the efficiency of the compressor dispersive elements (gratings) for X-ray radiation.

Another idea for compressing the $\sim 100$ ps pulses of the TGLS is based on the reflection on a mirror surface moving at a relativistic velocity. In this case the relativistic Doppler effect results in the frequency increase and decrease of the pulse duration by a factor of

$$
\Gamma=\frac{1+v / c}{1-v / c},
$$

where $v$ is the mirror velocity and $c$ is the speed of light in a vacuum. A relativistic mirror can be created by propagation of a high-intensity laser pulse in a gas of solid medium which is ionized by the laser. Reflection of electromagnetic waves on a moving ionization front has been discussed for a long time

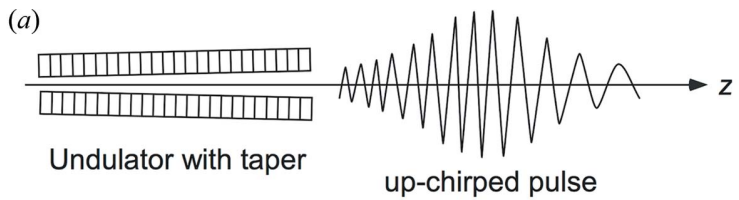

(b)

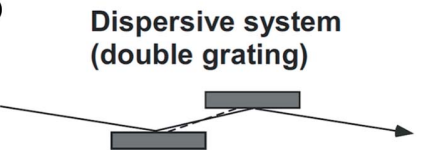

Figure 15

X-ray pulse compression scheme. (a) Up-chirped X-ray pulses are radiated through the undulator with tapered gap. $(b)$ Double grating dispersion system for compression of the chirped X-ray pulses. Reprinted with permission from Fujiki et al. (2009), Copyright (2009) by the American Physical Society.
(Lampe et al., 1978). Recently the first experimental demonstration of dense relativistic electron mirrors created by the interaction of a high-intensity laser pulse with a free-standing nanometer-scale thin foil was reported (Kiefer et al., 2013). It was shown that reflection on this mirror shifts the frequency of a counter-propagating laser pulse coherently from the infrared to the extreme ultraviolet region. Reflection on a relativistic mirror can be considered as a coherent backward Thomson scattering. Generation of X-ray pulses via scattering of a powerful laser light on a relativistic electron bunch was proposed about 50 years ago (Arutyunian \& Tumanian, 1963). Many experiments have been performed in a counter-propagating beam geometry. In this case the X-ray pulse duration is approximately equal to the duration of the electron bunch. In 1994 a novel geometry was proposed ( $90^{\circ}$ Thomson geometry) (Kim et al., 1994). In this case the X-ray pulse duration is determined by the transverse rather by the longitudinal electron bunch size. The first experimental demonstration of femtosecond X-ray pulse generation by this technique was in 1996 (Schoenlein et al., 1996). The main disadvantage of this source is very low brightness $\left(\sim 2 \times 10^{7}\right.$ photons $\mathrm{mm}^{-2}$ $\operatorname{mrad}^{-2} \mathrm{~s}^{-2}$ in a $\sim 15 \%$ bandwidth). Higher brightness could be achieved via coherent backwards Thomson scattering on a relativistic mirror (Kiefer et al., 2013; Schoenlein et al., 1996).

\section{TGLS operation in the era of free-electron lasers}

With the advent of the intense FEL source the legitimation for continued operation of TGLS X-ray sources could be put into question. From a scientific point of view, however, there exist several reasons why the established X-ray synchrotron facilities are certainly going to maintain a valuable tool for scientists for the years to come. Indeed, a large class of timeresolved experiments do not need the ultrashort ( $<50 \mathrm{fs}$ ) nor the ultrabright X-ray pulses provided by FELs but can deal with somewhat weaker and longer (0.1-10 ps) pulses. Spacecharge sensitive experiments, for example, require highest possible repetition rate rather than highest possible peak power. Developments towards shorter X-ray pulses from $\mathrm{MHz}$ TGLS will therefore be important steps for future cuttingedge science opportunities, as these sources are complementary in pulse duration and repetition rate to the presently available FEL facilities. We are convinced that with the anticipated technological evolutions towards low emittance and shorter pulses the storage-ring-based synchrotrons will enable competitive science also in the future. More than 50 synchrotrons are operated worldwide and offer scientists a large diversity of established X-ray diagnostics at several hundreds of beamlines. We mention that the currently fast progressing laser technology based on OPCPA and fiber technology (Mourou et al., 2013) towards higher average power could help to further increase the performance of the already established slicing technology presented in $\$ 2$ by increasing the repetition rate to tens of $\mathrm{kHz}$. This will enable significantly faster data acquisition and improved performance of the current slicing sources, which offer virtually jitter-free pump-probe conditions. 
The recent advent of high-gain FELs offering femtosecond radiation at unprecedented brilliance has undoubtedly enabled new science (Rohringer et al., 2012; Young et al., 2010b; Chapman et al., 2011). However, the challenge of the large arrival time jitter present at SASE-FELs is currently a formidable hurdle for reaching the ultimate temporal resolution of these machines. Two different strategies are currently being considered to overcome this limitation. The first one aims at measuring for each X-ray pulse the arrival time in relation to the optical pump pulse (Hartmann et al., 2014; Juranić et al., 2014; Harmand et al., 2013). This set of information is used to bin the experimental data as a function of the arrival time. The currently most promising technological solutions are based on monitoring ultrafast changes in reflection of the optical pulse in a sample pumped by the X-ray pulse or by streaking photoelectrons produced by the FEL with the optical pump laser (or a sub-harmonic thereof). Both methods have shown their potential to identify the arrival time jitter with an accuracy of approximately $10 \mathrm{fs}$ or less and future progress may lead to a further improvement to a few femtoseconds. The second approach employs an optical laser (or a higher harmonic thereof) as seed for coupling the longitudinal FEL modes coherently during the lasing process (Ackermann et al., 2013; Lambert et al., 2008; Allaria et al., 2012). Seeding requires a coherent X-ray source which is powerful enough to overcome the SASE noise level by about two orders of magnitude. The seeding concept in the ultraviolet is now routinely used at the FEL user facility in Italy (Fermi@Elettra) where excellent time-of-arrival stability could be demonstrated (Allaria et al., 2012). But the current challenge, however, is FEL seeding at shorter wavelengths $(\ll 10 \mathrm{~nm})$. At such short wavelengths, state-of-the-art laser sources based on high-order harmonic generation do not presently offer sufficient peak power to overcome the SASE shot noise. Further developments in high peak power HHG sources are thus required for exploring the seeding scheme at wavelengths $<10 \mathrm{~nm}$. While the required powerful laser systems will match the present repetition rate of FELs (10$100 \mathrm{~Hz}$ ), they will be unable to cope with significantly larger repetition rates in the near future.

\section{Conclusions}

Different techniques for X-ray pulse shortening at TGLS have been reviewed. Laser slicing is the most elaborate and established technique for obtaining $\sim 100$ fs $\mathrm{X}$-ray pulses from third-generation synchrotron light sources. Whereas the peak brightnesses of these femtosecond X-ray pulses are significantly lower than what FELs provide, it is the high repetition rate $(\mathrm{kHz})$, the intrinsically low timing jitter and accessibility which maintains the source attractive for users also in the future. Several types of X-ray switches were proposed for shortening X-ray pulses from TGLS. Until now only one type of ultrafast X-ray switch was tested experimentally at a TGLS, based on dynamics of coherent acoustic phonons in a photoexcited thin film. Estimations indicate that even an 'ideal' $\mathrm{X}$-ray switch hardly surpasses the performance of the laser slicing technique in view of average and peak brightness. Subpicosecond X-ray pulses with higher brightness could be achieved by means of a temporal compression of TGLS X-ray pulses. However, an experimental realisation of X-ray pulse temporal compression still needs to be experimentally demonstrated. Elaborating pulse compression schemes at TGLS also in the future is justified by the large user community and the trend towards shorter pulses while maintaining the high $(\mathrm{MHz})$ repetition rate offered by these largescale facilities. Such schemes are expected to bridge the gap between X-ray pulse characteristics provided by standard TGLS (30-100 ps) and FELs (1-100 fs) which will enable new science opportunities in the future.

\section{Acknowledgements}

We would like to acknowledge fruitful discussions with SwissFEL and SLS personnel and partial financial support from the Swiss National Science Foundation (PP00P2_150732) and COST (MP1203, SERI grant no C13.016). CPH acknowledges association to NCCR-MUST.

\section{References}

Abo-Bakr, M., Feikes, J., Holldack, K., Kuske, P., Peatman, W. B., Schade, U., Wüstefeld, G. \& Hübers, H.-W. (2003). Phys. Rev. Lett. 90, 094801.

Abo-Bakr, M., Feikes, J., Holldack, K., Wüstefeld, G. \& Hübers, H. W. (2002). Phys. Rev. Lett. 88, 254801.

Ackermann, S. et al. (2013). Phys. Rev. Lett. 111, 114801.

Allaria, E. et al. (2012). Nat. Photon. 6, 699-704.

Arutyunian, F. R. \& Tumanian, V. A. (1963). Phys. Lett. 4, 176-178.

Beaud, P., Johnson, S. L., Streun, A., Abela, R., Abramsohn, D., Grolimund, D., Krasniqi, F., Schmidt, T., Schlott, V. \& Ingold, G. (2007). Phys. Rev. Lett. 99, 174801.

Bergead, N., López-Flores, V., Halté, V., Hehn, M., Stamm, C., Pontius, N., Beaurepaire, E. \& Boeglin, C. (2014). Nat. Commun. 5, 3466.

Boeglin, C., Beaurepaire, E., Halté, V., López-Flores, V., Stamm, C., Pontius, N., Dürr, H. A. \& Bigot, J. Y. (2010). Nature (London), 465, 458-461.

Bressler, C., Milne, C., Pham, V.-T., ElNahhas, A., van der Veen, R. M., Gawelda, W., Johnson, S., Beaud, P., Grolimund, D., Kaiser, M., Borca, C. N., Ingold, G., Abela, R. \& Chergui, M. (2009). Science, 323, 489-492.

Bucksbaum, P. H. \& Merlin, R. (1999). Solid State Commun. 111, 535539.

Buth, C., Santra, R. \& Young, L. (2007). Phys. Rev. Lett. 98, 253001.

Byrd, J. M., Hao, Z., Martin, M. C., Robin, D. S., Sannibale, F., Schoenlein, R, W., Zholents, A. A. \& Zolotorev, M. S. (2006). Phys. Rev. Lett. 96, 164801.

Cavalleri, A., Wall, S., Simpson, C., Statz, E., Ward, D. W., Nelson, K. A., Rini, M. \& Schoenlein, R. W. (2006). Nature (London), 442, 664-666.

Chapman, H. et al. (2011). Nature (London), 470, 73-77.

Eriksson, M., van der Veen, J. F. \& Quitmann, C. (2014). J. Synchrotron Rad. 21, 837-842.

Eschenlohr, A., Battiato, M., Maldonado, P., Pontius, N., Kachel, T., Holldack, K., Mitzner, R., Föhlisch, A., Oppeneer, P. M. \& Stamm, C. (2013). Nat. Mater. 12, 332-336.

Freund, I. \& Levine, B. F. (1970). Phys. Rev. Lett. 25, 1241-1245.

Frühling, U., Wieland, M., Gensch, M., Gebert, T., Schütte, B., Krikunova, M., Kalms, R., Budzyn, F., Grimm, O., Rossbach, J., Plönjes, E. \& Drescher, M. (2009). Nat. Photon. 3, 523-528. 
Fujiki, S., Tsuchiya, K., Ishikawa, J., Okuma, H., Miyahara, T., Sasaki, H., Shioya, T., Obina, T. \& Yamamoto, S. (2009). Phys. Rev. A, 80, 063804.

Gaal, P., Schick, D., Herzog, M., Bojahr, A., Shayduk, R., Goldshteyn, J., Leitenberger, W., Vrejoiu, I., Khakhulin, D., Wulff, M. \& Bargheer, M. (2014). J. Synchrotron Rad. 21, 380-385.

Gaal, P., Schick, D., Herzog, M., Bojahr, A., Shayduk, R., Goldshteyn, J., Navirian, H. A., Leitenberger, W., Vrejoiu, I., Khakhulin, D., Wulff, M. \& Bargheer, M. (2012). Appl. Phys. Lett. 101, 243106.

Glover, T. E. Fritz, D. M., Cammarata, M., Allison, T. K., Coh, S., Feldkamp, J. M., Lemke, H., Zhu, D., Feng, Y., Coffee, R. N., Fuchs, M., Ghimire, S., Chen, J., Shwartz, S., Reis, D. A., Harris, S. E. \& Hastings, J. B. (2012). Nature (London), 488, 603-608.

Glover, T. E., Hertlein, M. P., Southworth, S. H., Allison, T. K., van Tilborg, J., Kanter, E. P., Krässig, B., Varma, H. R., Rude, B., Santra, R., Belkacem, A. \& Young, L. (2010). Nat. Phys. 6, 69-74.

Grigoriev, A., Do, D.-H., Kim, D. M., Eom, C.-B., Evans, P. G., Adams, B. \& Dufresne, E. M. (2006). Appl. Phys. Lett. 89, 021109.

Harmand, M., Coffee, R., Bionta, M. R., Chollet, M., French, D., Zhu, D., Fritz, D. M., Lemke, H. T., Medvedev, N., Ziaja, B., Toleikis, S. \& Cammarata, M. (2013). Nat. Photon. 7, 215-218.

Hartmann, N., Helml, W., Galler, A., Bionta, M. R., Grünert, J. L., Molodtsov, S., Ferguson, K. R., Schorb, S., Swiggers, M. L., Carron, S., Bostedt, C., Castagna, J.-C., Bozek, J., Glownia, J. M., Kane, D. J., Fry, A. R., White, W. E., Hauri, C. P., Feurer, T. \& Coffee, R. N. (2014). Nat. Photon. 8, 706-709.

He, A., Willeke, F. \& Yu, L. H. (2014). Phys. Rev. ST-AB, 17, 040701. Hemsing, E., Stupakov, G., Xiang, D. \& Zholents, A. (2014). Rev. Mod. Phys. 86, 897-941.

Herzog, M., Leitenberger, W., Shayduk, R., van der Veen, R. M., Milne, C. J., Johnson, S. L., Vrejoiu, I., Alexe, M., Hesse, D. \& Bargheer, M. (2010). Appl. Phys. Lett. 96, 161906.

Hettel, R. (2014). J. Synchrotron Rad. 21, 843-855.

Holldack, K. et al. (2014). J. Synchrotron Rad. 21, 1090-1104.

Holldack, K., Khan, S., Mitzner, R. \& Quast, T. (2006). Phys. Rev. Lett. 96, 054801.

Huang, X., Rabedeau, T. \& Safranek, J. (2014). J. Synchrotron Rad. 21, 961-967.

Huse, N., Cho, H., Hong, K., Jamula, L., de Groot, F. M. F., Kim, T. K., McCusker, J. K. \& Schoenlein, R. W. (2011). J. Phys. Chem. Lett. 2, 880-884.

Johnson, S. L., Beaud, P., Milne, C. J., Krasniqi, F. S., Zijlstra, E. S., Garcia, M. E., Kaiser, M., Grolimund, D., Abela, R. \& Ingold, G. (2008). Phys. Rev. Lett. 100, 155501.

Juranić, P. N., Stepanov, A., Ischebeck, R., Schlott, V., Pradervand, C., Patthey, L., Radović, M., Gorgisyan, I., Rivkin, L., Hauri, C. P., Monoszlai, B., Ivanov, R., Peier, P., Liu, J., Togashi, T., Owada, S., Ogawa, K., Katayama, T., Yabashi, M. \& Abela, R. (2014). Opt. Express, 22, 30004.

Katayama, I., Aoki, H., Takeda, J., Shimosato, H., Ashida, M., Kinjo, R., Kawayama, I., Tonouchi, M., Nagai, M. \& Tanaka, K. (2012). Phys. Rev. Lett. 108, 097401.

Katoh, M. (1999). Jpn. J. Appl. Phys. 38, L547-L549.

Khan, S., Holldack, K., Kachel, T., Mitzner, R. \& Quast, T. (2006). Phys. Rev. Lett. 97, 074801.

Kiefer, D., Yeung, M., Dzelzainis, T., Foster, P. S., Rykovanov, S. G., Lewis, C. L. S., Marjoribanks, R. S., Ruhl, H., Habs, D., Schreiber, J., Zepf, M. \& Dromey, B. (2013). Nat. Commun. 4, 1763-1767.

Kim, K.-J., Chattopadhyay, S. \& Shank, C. V. (1994). Nucl. Instrum. Methods Phys. Res. A, 341, 351-354.

Lambert, G., Hara, T., Garzella, D., Tanikawa, T., Labat, M., Carre, B., Kitamura, H., Shintake, T., Bougeard, M., Inoue, S., Tanaka, Y., Salieres, P., Merdji, H., Chubar, O., Gobert, O., Tahara, K. \& Couprie, M.-E. (2008). Nat. Phys. 4, 296-300.

Lampe, M., Ott, E. \& Walker, J. (1978). Phys. Fluids, 21, 42-54.

Larsson, J., Heimann, P. A., Lindenberg, A. M., Schuck, P. J., Bucksbaum, P. H., Lee, R. W., Padmore, H. A., Wark, J. S. \&
Falcone, R. W. (1998). Appl. Phys. Mater. Sci. Process. 66, 587591.

Limborg, C. (1998). Proc. SPIE, 3451, 72-81.

Loh, Z.-H., Greene, C. H. \& Leone, S. R. (2008). Chem. Phys. 350, 7 13.

Mariager, S. O., Dornes, C., Johnson, J. A., Ferrer, A., Grübel, S., Huber, T., Caviezel, A., Johnson, S. L., Eichhorn, T., Jakob, G., Elmers, H. J., Beaud, P., Quitmann, C. \& Ingold, G. (2014). Phys. Rev. B, 90, 161103 .

Mobilio, S., Boscherini, F. \& Meneghini, C. (2015). Editors. Synchrotron Radiation: Basics, Methods and Application. Springer. Mourou, G., Brockslesby, B., Tajima, T. \& Limpert, J. (2013). Nat. Photon. 7, 258-261.

Nazarkin, A., Uschmann, I., Förster, E. \& Sauerbrey, R. (2004). Phys. Rev. Lett. 93, 207401

Radu, I., Vahaplar, K., Stamm, C., Kachel, T., Pontius, N., Dürr, H. A., Ostler, T. A., Barker, J., Evans, R. F. L., Chantrell, R. W., Tsukamoto, A., Itoh, A., Kirilyuk, A., Rasing, Th. \& Kimel, A. V. (2011). Nature (London), 472, 205-208.

Rettig, L., Mariager, S. O., Ferrer, A., Grübel, S., Johnson, J. A., Rittmann, J., Wolf, T., Johnson, S. L., Ingold, G., Beaud, P. \& Staub, U. (2015). Phys. Rev. Lett. 114, 067402.

Rohringer, N., Ryan, D., London, R. A., Purvis, M., Albert, F., Dunn, J., Bozek, J. D., Bostedt, C., Graf, A., Hill, R., Hau-Riege, S. P. \& Rocca, J. J. (2012). Nature (London), 481, 488-491.

Ruchert, C., Vicario, C. \& Hauri, C. P. (2013). Phys. Rev. Lett. 110, 123902.

Schoenlein, R. W., Chattopadhyay, S., Chong, H. H. W., Glover, T. E., Heimann, P. A., Shank, C. V., Zholents, A. A. \& Zolotorev, M. S. (2000). Science, 287, 2237-2240.

Schoenlein, R. W., Leemans, W. P., Chin, A. H., Volfbeyn, P., Glover, T. E., Balling, P., Zolotorev, M., Kim, K.-J., Chattopadhyay, S. \& Shank, C. V. (1996). Science, 274, 236-238.

Shalaby, M. \& Hauri, C. P. (2015). Nat. Commun., 6, 5976.

Sheppard, J. M. H., Sondhauss, P., Merlin, R., Bucksbaum, P., Lee, R. W. \& Wark, J. S. (2005). Solid State Commun. 136, 181-185.

Stamm, C., Kachel, T., Pontius, N., Mitzner, R., Quast, T., Holldack, K., Khan, S., Lupulescu, C., Aziz, E. F., Wietstruk, M., Dürr, H. A. \& Eberhardt, W. (2007). Nat. Mater. 6, 740-743.

Stepanov, A. G., Henin, S., Petit, Y., Bonacina, L., Kasparian, J. \& Wolf, J.-P. (2010). Appl. Phys. B, 101, 11-14.

Vicario, C., Jazbinsek, M., Ovchinnikov, A. V., Chefonov, O. V., Ashitkov, S. I., Agranat, M. B. \& Hauri, C. P. (2015). Opt. Express, 23, 4573-4580.

Vicario, C., Monoszlai, B. \& Hauri, C. P. (2014b). Phys. Rev. Lett. 112, 213901.

Vicario, C., Ovchinnikov, A. V., Ashitkov, A. I., Agranat, M. B., Fortov, V. E. \& Hauri, C. P. (2014a). Opt. Lett. 39, 66326635.

Wietstruk, M., Melnikov, A., Stamm, C., Kachel, T., Pontius, N., Sultan, M., Gahl, C., Weinelt, M., Dürr, H. A. \& Bovensiepen, U. (2011). Phys. Rev. Lett. 106, 127401.

Wuestenfeld, G., Jankowiak, A., Knoblauch, J. \& Ries, M. (2011). Proceedings of IPAC2011, San Sebastian, Spain. THPC014.

Young, L., Buth, C., Dunford, R. W., Ho, P., Kanter, E. P., Krássig, B., Peterson, E. R., Rohringer, N., Santra, R. \& Southworth, S. H. (2010a). Rev. Mex. Fis. 56, 11-17.

Young, L., Kanter, E. P., Krässig, B., Li, Y., March, Y., Pratt, S. T., Santra, R., Southworth, R., Rohringer, N., DiMauro, N., Doumy, G., Roedig, G., Berrah, N., Fang, L., Hoener, M., Bucksbaum, M., Cryan, J. P., Ghimire, S., Glownia, S., Reis, D. A., Bozek, J. D., Bostedt, C. \& Messerschmidt, M. (2010b). Nature (London), 466, $56-61$.

Zholents, A., Heimann, P., Zolotorev, M. \& Byrd, J. (1999). Nucl. Instrum. Methods Phys. Res. A, 425, 385-389.

Zholents, A. A. \& Zolotorev, M. S. (1996). Phys. Rev. Lett. 76, $912-$ 915. 